\title{
Oxidative Stress (Malondialdehyde) in Adults Beta-Thalassemia Major and Intermedia: Comparison Between Before and After Blood Transfusion and Its Correlation with Iron Overload
}

\author{
Tubagus Djumhana \\ Atmakusuma (D' \\ Intan Russiana Nasution ${ }^{2}$ \\ Noorwati Sutandyo ${ }^{3}$ \\ 'Division of Hematology-Medical \\ Oncology, Department of Internal \\ Medicine, Dr. Cipto Mangunkusumo \\ General Hospital/Faculty of Medicine \\ Universitas Indonesia, Jakarta, Indonesia; \\ ${ }^{2}$ Division of Hematology-Medical \\ Oncology, Department of Internal \\ Medicine, Gatot Soebroto Army Hospital \\ Jakarta/Faculty of Medicine Universitas \\ Indonesia, Jakarta, Indonesia; ${ }^{3}$ Division of \\ Hematology-Medical Oncology, \\ Department of Internal Medicine, \\ Dharmais National Cancer Hospital \\ Jakarta/Faculty of Medicine Universitas \\ Indonesia, Jakarta, Indonesia
}

Background: Iron overload is a major problem in both transfusion-dependent (TDT) and non-transfusion-dependent thalassemia (NTDT). It has been known to increase oxidative stress. Meanwhile, blood transfusion as main therapy for thalassemia increases iron overload. One of the markers of oxidative stress is malondialdehyde (MDA). This study aims to provide data on MDA levels in adult thalassemia patients, and to compare the levels before and after transfusion in patients with TDT and NTDT.

Methods: This is a cross-sectional, pre-post study in adult patients with thalassemia major and intermedia that received blood transfusion with or without iron-chelating agents in Cipto Mangunkusumo Hospital. Blood samples were taken immediately before the transfusion and one day after. Serum ferritin (SF) assays were conducted by electrochemiluminescence immunoassay method, while transferrin saturation (TS) was calculated by dividing serum iron by the binding capacity. Subsequently, plasma MDA levels assays were performed using the Wills method, and data analysis was conducted using the $t$-test/Mann-Whitney and Pearson/Spearman correlation test, depending on the data distribution.

Results: The 63 respondents recruited consist of 51 TDT and 12 NTDT patients, and their median plasma MDA level before and after transfusion was $0.49 \mu \mathrm{mol} / \mathrm{L}$ and $0.45 \mu \mathrm{mol} / \mathrm{L}$, respectively. Before transfusion, there was no correlation between SF and MDA, and TS and MDA levels. After the transfusion, there was no correlation between, SF and MDA, or TS and MDA levels.

Conclusion: There is no significant difference in MDA levels before and after transfusion. Although blood transfusion increases the iron load in thalassemia patients, there was no increase in median MDA level after transfusion. Meanwhile, there was no correlation between markers of iron overload and MDA level in thalassemia patients both before and after transfusion.

Keywords: iron overload, malondialdehyde, oxidative stress, non-transfusion dependent thalassemia, transfusion-dependent thalassemia

\section{Background}

Thalassemia is a hereditary disease caused by a defect in globin synthesis. This results in erythrocyte membrane fluidity. Thalassemia allows erythrocytes to be broken down easily, thereby lowering the level of hemoglobin in the blood. This phenomenon will result in chronic non-immune hemolytic anemia. ${ }^{1-3}$ Indonesia is 
one of the countries with a high prevalence number of thalassemia. Data from the Thalassemia Center, Cipto Mangunkusumo Hospital (RSCM) in 2016, showed that there were 9.031 patients with thalassemia major, and this number is expected to be higher since we have high prevalence of thalassemia gene carriers which are asymptomatic and have not conducted any premarital screening. ${ }^{4}$

Thalassemia major (TM) is characterized by severe anemia requiring red blood cell transfusions regularly for growth and survival. ${ }^{5}$ Meanwhile, thalassemia intermedia (TI) has a clinical spectrum or phenotype which varies widely from requiring regular blood transfusions (transfusion-dependent thalassemia/TDT) to not requiring regular transfusions (non-transfusion-dependent thalassemia/ NTDT). Generally, complications of excess iron load in TM are due to transfusion of red blood cells, which may result in organ toxicity caused by free radicals, reactive oxygen species (ROS). People with TI are also at risk of iron overload due to the increased absorption of iron from the digestive tract. ${ }^{6}$

Excessive iron overload forms non-transferrin-bound iron (NTBI) with the most toxic labile plasma iron (LPI) fraction. However, the NTBI examination has not been standardized and does not yet exist in Indonesia. Transferrin saturation (TS) can be used as a surrogate marker for NTBI examination. ${ }^{7}$ Furthermore, NTBI ferrous iron (Fe II) induces the increased production of ROS, especially hydroxyl radicals $(\mathrm{OH} \bullet)$ by the Fenton and Haber Weiss reaction. ${ }^{8,9}$ The formed ROS will cause lipid peroxidation (PL) cell membrane with one of the products namely malondialdehyde (MDA), which is toxic and mutagenic. MDA is used as a biomarker of oxidative stress examination. ${ }^{10}$

Existing study on oxidative stress shows conflicting results. Some studies in pediatric and adults with betathalassemia found correlation between SF and MDA levels. ${ }^{11,12}$ However, different results were obtained by Al-Hakeim et $\mathrm{al}^{13}$ and Gunarsih et al, who found no correlation between SF and MDA. Meanwhile, results from the study by Cighetti et $\mathrm{al}^{14}$ showed a correlation between NTBI and MDA. On the contrary, Al-Hakeim, et $\mathrm{al}^{13}$ and Walter et $\mathrm{al}^{15}$ did not obtain the correlation between NTBI with MDA. These warrant further study, especially in adult thalassemia patients since most studies were conducted in pediatric population.

Regular blood transfusion therapy of thalassemia causes iron overload that will induce the formation of ROS. However, there is no published data about the effect of transfusion on changes in oxidative stress. MDA was selected as a marker of oxidative stress because the sampling and examination techniques are relatively easy. Therefore, this study aims to measure the change of MDA levels as a biomarker of oxidative stress related to the provision of blood transfusion therapy. Furthermore, we also aim to analyze the relationship of iron overload and MDA level in thalassemia patients both before and after transfusion.

\section{Methods}

\section{Population}

The targeted population is adult patients with beta-thalassemia in RSCM at the Thalassemia and Hematology Polyclinic, Department of Internal Medicine. They received blood transfusions either regularly (thalassemia major or TDT-phenotype thalassemia intermedia) or as required (thalassemia minor or NTDT-phenotype thalassemia intermedia). Furthermore, the selected subjects were a those who met the inclusion criteria of the study.

\section{Inclusion Criteria}

The subjects recruited were aged 18 years or more and were diagnosed with beta-thalassemia with high-performance liquid chromatography (HPLC) or microcapillary method. They were willing to participate in the study after signing a written consent form. Furthermore, the subjects that fulfilled inclusion criteria were categorized into TDT and NTDT groups.

\section{Exclusion Criteria}

The patients who experienced acute medical conditions (heart failure, liver failure), acute inflammation (temperature $>38^{\circ} \mathrm{C}$ ), chronic inflammation or infection, pregnant woman, and malignancy were excluded.

\section{Recruitment of Subjects}

The subjects were recruited consecutively from at the Thalassemia and Hematology Polyclinic, Department of Internal Medicine, Cipto Mangunkusumo National Referral Hospital, Jakarta. Furthermore, the demographic data and the volume of blood and frequency of red cell transfusions in the past year were recorded from the medical records of patients, and they were categorized into TDT and NTDT. 


\section{Laboratory Procedures}

The patients underwent venous blood sampling twice, ie, before and after blood transfusion. Subsequently, blood samples were taken each as much as $12 \mathrm{~mL}$ and $5 \mathrm{~mL}$, and the test for the value of hemoglobin, serum ferritin, hsCRP, transferrin saturation, and malondialdehyde (MDA) was then conducted.

Measurement of MDA was conducted by spectrophotometric method (Wills) using the principle of the reaction with thiobarbituric acid. This procedure uses $3 \mathrm{~mL}$ of blood serum mixed with trichloroacetic acid and was centrifuged to form a supernatant. Furthermore, the supernatant or protein deposits were added with acetic acid, thiobarbituric acid, and n-butanol to produce colors. The color formed is read at a wavelength of $532 \mathrm{~nm}$ by spectrophotometry.

\section{Ethics}

This study has been reviewed and approved by The Ethics Committee of the Faculty of Medicine, Universitas Indonesia, and the subjects provided appropriate consent to participate. This study was conducted following the declaration of Helsinki, and all participants were informed about the purpose of the study.

\section{Data Analysis}

The collected data were processed using SPSS 20 for Windows. Data analysis was conducted using the $t$-test/ Mann-Whitney and Pearson/Spearman correlation test, depending on the data distribution.

\section{Results}

\section{Demographic Data}

A total of 63 adult patients with beta-thalassemia were recruited during this study period, and it consisted of 51 TDT and 12 NTDT patients. There were 37 females (58\%) and 26 males (42\%) subjects, and the median age was 25 years with a range from 18 to 67 years. Furthermore, the number of patients with beta-thalassemia was 21 (33\%) and beta-thalassemia/HbE was 42 people $(67 \%)$. Clinical characteristics of subjects with TDT were almost equal to patients with NTDT in terms of the gender ratio, age, hemoglobin level and body mass index (BMI). Patients with TDT received much larger and more frequent blood transfusions than NTDT (Table 1).

\section{Measurement of MDA Plasma}

The median plasma MDA level on the subjects before transfusion was $0.49 \mu \mathrm{mol} / \mathrm{L}$ with the lowest and the highest value of $0.21 \mu \mathrm{mol} / \mathrm{L}$ and $1.33 \mu \mathrm{mol} / \mathrm{L}$. Meanwhile, the median plasma MDA level after transfusion was 0.45 $\mu \mathrm{mol} / \mathrm{L}$ with the lowest and highest value of $0.14 \mu \mathrm{mol} /$ $\mathrm{L}$ and $1.49 \mu \mathrm{mol} / \mathrm{L}$, respectively.

\section{Differences in Plasma MDA Levels Between TDT and NTDT Groups}

There were no significant differences in plasma MDA levels before and after transfusion in adult patients with beta-thalassemia TDT and NTDT (Table 2).

\section{Plasma MDA Level in Adult Patients with Beta-Thalassemia TDT and NTDT Between Before and After Transfusion}

The analysis showed that there was no significant difference in the change (delta) median plasma MDA levels before and after transfusion in adult patients with betathalassemia TDT and NTDT (Table 3).

Correlation Between Serum Ferritin and Transferrin Saturation with Plasma MDA Levels Before and After Transfusion in Adult Patients with Beta-Thalassemia TDT and NTDT

Before transfusion, there was no correlation between SF and MDA, and TS and MDA levels. After the transfusion, there was no correlation between, SF and MDA, or TS and MDA levels in adult patients with beta-thalassemia (TDT and NTDT) (Table 4).

Although Pearson correlation test showed significant correlation between SF and MDA before transfusion, the data distribution was not normal. Hence, Spearman correlation was used and resulted in no correlation.

\section{Changes in Plasma MDA}

The changes in plasma MDA levels in these two groups can be seen in Table 5 and Figure 1.

\section{Discussion}

A total of 63 patients were included in this study, consisting of 51 TDT and 12 NTDT patients with a median age of 25 years old. Furthermore, the median levels of MDA were 0.49 $(\min 0.21 ; \max 1.33) \mu \mathrm{mol} / \mathrm{L}$ and were not significantly 
Table I Characteristics of the Subjects Study According TDT and NTDT Groups Before Transfusion

\begin{tabular}{|c|c|c|c|}
\hline \multirow{2}{*}{ Characteristics } & \multicolumn{2}{|c|}{ Adult Beta Thalassemia } & \multirow{2}{*}{$\begin{array}{c}\text { Total (TDT+NTDT) } \\
(n=63)\end{array}$} \\
\hline & $\begin{array}{c}\text { TDT } \\
(n=5 I)\end{array}$ & $\begin{array}{c}\text { NTDT } \\
(n=12)\end{array}$ & \\
\hline \multicolumn{4}{|l|}{ Gender, n (\%) } \\
\hline Man & $21(41.2)$ & $5(41.7)$ & $26(41.3)$ \\
\hline Woman & $30(58.8)$ & $7(58.3)$ & $37(58.7)$ \\
\hline Age (years), median (min-max) & $25(18-67)$ & $25(19-66)$ & $25(18-67)$ \\
\hline \multicolumn{4}{|l|}{ Diagnosis genotype, $n(\%)$} \\
\hline Beta thalassemia & $15(29.4)$ & $6(50.0)$ & $21(33.3)$ \\
\hline Beta thalassemia/HBE & $36(70.6)$ & $6(50.0)$ & $42(66.7)$ \\
\hline BMI $\left(\mathrm{kg} / \mathrm{m}^{2}\right)$, mean $(\mathrm{SD})$ & $18.7(2)$ & $20(2.9)$ & $18.97(2.3)$ \\
\hline \multicolumn{4}{|l|}{ Iron chelation, n (\%) } \\
\hline Deferiprone & $40(78.4)$ & $6(50)$ & $46(73.0)$ \\
\hline Deferasirox & $9(17.6)$ & $2(16.7)$ & II (I7.5) \\
\hline Deferoxamine & I (2) & - & $\mathrm{I}(1.6)$ \\
\hline No chelating agent & I (2) & $4(33.3)$ & $5(7.9)$ \\
\hline Transferrin saturation (\%), median (min-max) & $81(30-100)$ & $85.5(28-94)$ & $82(2-100)$ \\
\hline$\geq 50 \%, \mathrm{n}(\%)$ & $37(72.5)$ & II (91.7) & $48(76.2)$ \\
\hline$<50 \%, \mathrm{n}(\%)$ & $14(27.5)$ & I (8.3) & $15(23.8)$ \\
\hline The mean serum ferritin in a year $(\mathrm{ng} / \mathrm{mL})$, median (min-max) & $3,094(344-14,012)$ & $3,863(963-5,944)$ & $3,136(344-14,012)$ \\
\hline$\geq 1000$ ng/mL, n (\%) & $47(92.2)$ & II (91.7) & $58(92.1)$ \\
\hline$<1000$ ng/mL, n (\%) & $4(7.8)$ & I (8.3) & $5(7.9)$ \\
\hline$\geq 2500$ ng/mL, n (\%) & $28(54.9)$ & $8(66.7)$ & $36(57.1)$ \\
\hline$<2500$ ng/mL, n (\%) & $23(45.1)$ & $4(33.3)$ & $27(42.9)$ \\
\hline MDA plasma before transfusion ( $\mu$ mol/L), mean $(S D)$ & $0.55(0.23)$ & $0.44(0.11)$ & $0.49(0.21-1.33)$ \\
\hline MDA plasma after transfusion ( $\mu$ mol/L), median (min-max) & $0.47(0.1-1.2)$ & $0.41(0.3-1.49)$ & $0.45(0.14-1.49)$ \\
\hline $\mathrm{Hb}$ before transfusion $(\mathrm{g} / \mathrm{dl})$, median (min-max) & $7.6(4.90-10.70)$ & $8.05(5.70-9.80)$ & $7.66(1.34)$ \\
\hline Volume of blood transfused during research $(\mathrm{mL})$, median (min-max) & $168(24-468)$ & $156(60-528)$ & $546(220-1,233)$ \\
\hline Volume of blood transfusions in the past year $(\mathrm{mL})$, median (min-max) & $5,965(1,918-13,096)$ & $2,124(280-4,847)$ & $4,847(280-13,096)$ \\
\hline Frequency of transfusions in a year, mean (SD) & $10(4)$ & $4(2)$ & $8.84(4.29)$ \\
\hline
\end{tabular}

Abbreviations: TDT, transfusion-dependent thalassemia; NTDT, non-transfusion-dependent thalassemia; SD, standard deviation; BMI, body mass index; MDA, malondialdehyde; $\mathrm{Hb}$, hemoglobin.

Table 2 Plasma MDA Levels Before and After Transfusion in Adult Patients with Beta-Thalassemia TDT and NTDT

\begin{tabular}{|l|c|c|c|}
\hline \multirow{2}{*}{ Variables } & \multicolumn{2}{|c|}{ Adult Beta Thalassemia (N=63) } & \multirow{2}{*}{ P $^{*}$} \\
\cline { 2 - 4 } & TDT & NTDT & \\
\hline MDA plasma before transfusion $(\mu \mathrm{mol} / \mathrm{L})$, mean $(\mathrm{SD})$ & $0.55(0.23)$ & $0.44(0.1 \mathrm{I})$ & 0.100 \\
MDA plasma after transfusion $(\mu \mathrm{mol} / \mathrm{L})$, median $(\min -\max )$ & $0.49(0.14-\mathrm{l} .20)$ & $0.38(0.3 \mathrm{I}-\mathrm{I} .49)$ & 0.299 \\
\hline
\end{tabular}

Note: *Mann-Whitney.

Table 3 Change (Delta) Plasma MDA Levels in Adult Patients with Beta-Thalassemia TDT and NTDT Between Before and After Transfusion

\begin{tabular}{|l|c|c|}
\hline Variables & Adult Beta-Thalassemia (TDT + NTDT) N = 57 & $\mathbf{P}$ \\
\hline Change (delta) plasma MDA $(\mu \mathrm{mol} / \mathrm{L})$, median $(\min -\mathrm{max})$ & $-0.007([-0.069]-0.055)$ & 0.820 \\
\hline
\end{tabular}


Table 4 The Correlation Between Serum Ferritin and Transferrin Saturation with Plasma MDA Levels Before and After Transfusion

\begin{tabular}{|l|c|c|c|c|}
\hline \multirow{2}{*}{ Variables } & \multicolumn{2}{|c|}{ Before Transfusion } & \multicolumn{2}{c|}{ After Transfusion } \\
\cline { 2 - 5 } & Pearson Correlation & Spearman Correlation & Pearson Correlation & Spearman Correlation \\
\hline Serum ferritin vs MDA & $r=0.256^{*}$ & $r=0.181$ & $r=0.107$ & $r=0.123$ \\
& $p=0.043$ & $p=0.156$ & $p=0.428$ & $p=0.361$ \\
\hline Transferrin saturation vs MDA & $r=0.192$ & $r=0.194$ & $r=0.077$ & $r=-0.090$ \\
& $p=0.138$ & $p=0.133$ & $p=0.578$ & $p=0.513$ \\
\hline
\end{tabular}

Note: *Pearson correlation test: $\mathrm{p}<0.05$.

Table 5 The Proportion of Subjects According to Changes of Plasma MDA Levels Before and After Transfusion in Adult Patients with Thalassemia Beta TDT and NTDT

\begin{tabular}{|l|c|c|c|}
\hline \multirow{2}{*}{ Changes in Plasma MDA } & \multicolumn{2}{|c|}{ Adult Beta Thalassemia } & \multirow{2}{*}{ Total } \\
\cline { 2 - 4 } & TDT, $\mathbf{n}(\%)$ & NTDT, $\mathbf{n}(\%)$ & $24(42.1)$ \\
\hline Increase & $19(39.6)$ & $5(55.6)$ & $1(1.8)$ \\
Permanent & $1(2.1)$ & $0(0)$ & $32(56.1)$ \\
Decline & $28(58.3)$ & $4(44.4)$ & \\
\hline
\end{tabular}

different between before and after transfusion. Differences in the results of plasma MDA levels with previous studies can be assumed to be caused by different subject characteristics. This includes the use of iron chelation therapy and antioxidant supplementation as well as the methods used in the measurement of MDA levels. Plasma MDA level tends to be increased in thalassemia and this reflects the oxidative stress status of the patients. ${ }^{16}$ Compared to healthy controls, patients with thalassemia show increased MDA levels. ${ }^{16,17}$ The increase in this lipid peroxidation product is considered to be closely related to the degree of oxidative stress as reported by Ozturk et al. ${ }^{18}$

The subjects used iron chelation therapy and antioxidant supplementation regularly. Several studies have been conducted on the effect of iron chelation and antioxidants on changes in MDA levels as a marker of oxidative stress.
Plasma MDA levels were decreased on the subject approaching the levels in healthy controls after receiving iron chelation therapy and antioxidants for 6-12 months. ${ }^{19,20}$ A study conducted by Nasseri, et $\mathrm{al}^{21}$ showed that administration of antioxidants in thalassemia patients decreases MDA levels. Therefore, plasma MDA levels obtained in this study were likely to be influenced by routine supplementation of antioxidants and iron chelation therapy.

Median levels of MDA was not significantly different in patients with TDT and NTDT ( 0.55 vs $0.44 \mu \mathrm{mol} / \mathrm{L} ; \mathrm{p}=0.100$; Mann-Whitney test). The NTDT group consists of patients with thalassemia that received blood transfusions, but not regularly with a mean frequency of 4 transfusions per year. On the contrary, TDT patients consist of beta-thalassemia major and TDT-phenotype thalassemia intermedia patients that require regular blood transfusions. However, this study
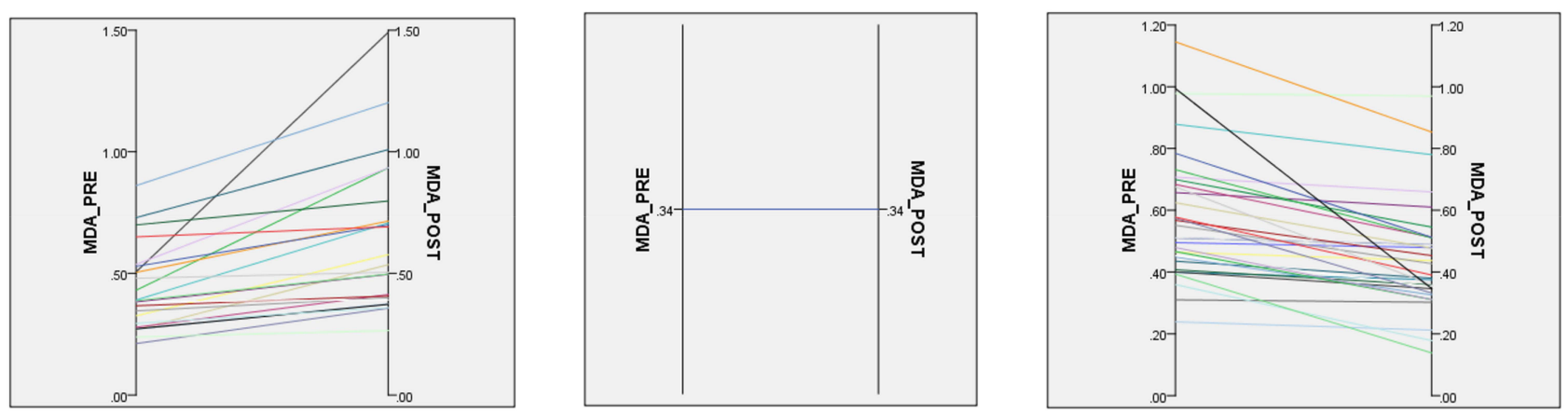

Figure I Line graph depicting the increase (left), stagnation (middle), and decrease (right) in MDA levels between study subjects, pre and post transfusion. 
shows that the difference of MDA levels between TDT and NTDT groups was not significant.

There was no correlation between SF and MDA levels before transfusion. Although Pearson test showed a significant weak correlation, since our data distribution was abnormal, nonparametric test should be used. Spearman correlation test showed that there was no correlation between SF and MDA as well as TS and MDA before and after transfusion.

Similar results have been reported Gunarsih et $\mathrm{al}^{1}$ in children with thalassemia major in the same center where our study was conducted. In their study, the correlation between serum ferritin and plasma MDA concentration was not obtained $(\mathrm{r}=0.147 ; \mathrm{p}=0.285)$. In addition, a study conducted by Al-Hakeim, et, a $1^{13}$ on 118 patients with betathalassemia major in children age 4-12 years also showed no correlation between serum ferritin levels of plasma MDA. On the contrary, another study by Cighetti et al ${ }^{14}$ on 21 patients with beta-thalassemia major age 19-30 years and 13 with beta-thalassemia intermedia $23-46$ years of age obtained a positive correlation between total MDA and NTBI ( $r=0.45 ; p=0.037)$. However, in our study, we found no correlation between total MDA and serum ferritin level.

Malondialdehyde (MDA) is a well-established marker for oxidative stress and cellular damage. Oxidative stress happens in many disease states such as cancer, chronic inflammation, and thalassemia. ${ }^{22}$ Oxidative stress is a result from the imbalance of oxidants and antioxidants. When the balance tip towards oxidants (ie, insufficient antioxidant activity), cellular damage occurs. Furthermore, the formation of reactive oxygen species (ROS) contributes to DNA damage, cellular injury, and even carcinogenesis. ${ }^{22}$ In thalassemia, iron overload plays a major role in mediating oxidative stress.

There was no previous study which correlated serum ferritin levels and plasma MDA in serial pattern before and after transfusion. In $58 \%$ of TDT patients, MDA levels decreased after transfusion. Meanwhile, in NTDT, 55.6\% had increased MDA levels. The absence of plasma MDA increase after transfusion in some subjects might be related to routine supplementation of antioxidants, which could attenuate the rise in MDA levels. A study by Ezzat, et $\mathrm{al}^{23}$ showed that patients with thalassemia have reduced antioxidant activity and vitamin $E$ levels. Therefore, routine supplementation of vitamin $\mathrm{E}$ or other antioxidants should be considered a rational approach. In subjects with increased MDA levels after transfusion, the supplementation of antioxidants may not be sufficient to suppress the oxidative stress damage. Therefore, antioxidants such as vitamin E can be used to attenuate this process. ${ }^{21}$ In this study, the majority of patients were routinely prescribed antioxidants.

In Islamic countries such as the Middle East and Indonesia, there is a practice of blood cupping called Alhijamah. Even though there is no data on the proportion of thalassemia patients that received this treatment, a lot of Indonesians follow the practice. Blood cupping is scientifically proven to reduce iron overload, serum ferritin, and oxidative stress. ${ }^{24}$ It removes MDA from circulating blood to reduce oxidative stress. ${ }^{25}$ Therefore, this method can be considered in select thalassemia patients, especially in those without thrombocytopenia due to hypersplenism. If thrombocytopenia has already occurred, blood cupping might induce massive blood loss which will increases the transfusional requirement.

Another concern of measuring plasma MDA level after transfusion is the possibility of interference from the donor's MDA. However, since leukodepleted or washedpacked red cells are only used for routine transfusion, the plasma content of MDA should be negligible.

Furthermore, the transferrin saturation did not correlate with plasma MDA levels either before or after transfusion. Transferrin saturation is a surrogate marker for NTBI. NTBI is the gold standard for measuring iron overload, although the examination is not available in Indonesia. Therefore, transferrin saturation was employed instead of NTBI, and there was no correlation between FS and TS with MDA levels. Therefore, further study should measure NTBI instead of FS and TS as markers of iron overload. In terms of oxidative stress, in addition of malondialdehyde, activity of other aldehydes can be measured by using assay for thiobarbituric acid reactive species. ${ }^{26}$

The sample size used is larger than similar studies conducted in Pakistan and Egypt. ${ }^{23,27}$ Moreover, the novelty of this study is the fact the MDA levels were measured pre- and post-transfusion. This is the first study that employed serial measurements of MDA levels. Limitation of this study is the limited number of participants due to the short study period and budget restrictions since MDA-level assay are not covered by national health insurance. Further studies involving a larger sample size is encouraged to confirm our findings. Moreover, it should involve patients with thalassemia major, intermedia, minor, and normal control group to compare the relationship between blood transfusion, iron overload, and oxidative stress. In addition, the subjects should be stratified based on baseline SF and TS to detect the difference in MDA levels. 


\section{Conclusion}

Median plasma MDA levels in adult beta-thalassemia major and intermedia before and after transfusion were $0.49 \mu \mathrm{mol} /$ $\mathrm{L}$ and $0.45 \mu \mathrm{mol} / \mathrm{L}$, respectively. There was no significant difference between MDA levels and between TDT and NTDT patients. Furthermore, there was no correlation between FS and TS with MDA before and after transfusion. The routine administration of antioxidants, especially vitamin E, might play a role in ameliorating MDA in thalassemia patients. Therefore, although blood transfusion increases iron load in thalassemia patients, there was no increase in median MDA level after transfusion.

\section{Disclosure}

The authors report no conflicts of interest for this work.

\section{References}

1. Gunarsih A, Amalia P, Boediman I. Variables associated with malondialdehyde level in patients with thalassemia major. Paediatr Indones. 2012;52:125-131. doi:10.14238/pi52.3.2012.125-31

2. Cappellini MD, Cohen A, Porter J, et al. Guidelines for the Management of Transfusion-Dependent Thalassemia. Thalassemia International Federation; 2014.

3. Gamberini MR, De Sanctis V, Gilli G. Hypogonadism, diabetes mellitus, hypothyroidism, hypoparathyroidism: incidence and prevalence related to iron overload and chelation therapy in patients with thalassaemia major followed from 1980 to 2007 in the Ferrara Centre. Pediatr Endocrinol Rev. 2008;6(Suppl 1):158-169.

4. Thalassemia Center Cipto Mangunkusumo Hospital. Thalassemia major patients data. Jakarta; 2016. [unpublished document].

5. Wood B, Molecular Higgs D. Basis of thalassemia syndromes. Disorders of erythropoiesis, erythrocytes and iron metabolism. Eur Sch Hematol. 2009;35:406

6. Taher AT, Vichinsky E, Musallam Ea K. Guidelines for the Management of Non-Transfusion Dependent Thalassemia (NTDT). Thalassemia International Federation; 2013.

7. Kohgo Y, Ikuta $\mathrm{K}$, Ohtake $\mathrm{T}$, et al. Body iron metabolism and pathophysiology of iron overload. Int J Hematol. 2008;88(1):7-15. doi:10.1007/s12185-008-0120-5

8. Hershko C. Pathogenesis and management of iron toxicity in thalassemia. Ann N Y Acad Sci. 2010;1202:1-9. doi:10.1111/j.17496632.2010.05544.x

9. Kalpravidh RW, Siritanaratkul N, Insain P, et al. Improvement in oxidative stress and antioxidant parameters in beta-thalassemia/Hb E patients treated with curcuminoids. Clin Biochem. 2010;43(4 5):424-429. doi:10.1016/j.clinbiochem.2009.10.057

10. Nielsen F, Mikkelsen BB, Nielsen JB, et al. Plasma malondialdehyde as biomarker for oxidative stress: reference interval and effects of life-style factors. Clin Chem. 1997;43(7):1209-1214. doi:10.1093/ clinchem/43.7.1209

11. Sengsuk C, Tangvarasittichai O, Chantanaskulwong $P$, et al. Association of iron overload with oxidative stress, hepatic damage and dyslipidemia in transfusion-dependent $\beta$-Thalassemia/ $\mathrm{HbE}$ patients. Indian J Clin Biochem. 2014;29(3):298-305. doi:10.1007/ s12291-013-0376-2
12. Naithani R, Chandra J, Bhattacharjee J, et al. Peroxidative stress and antioxidant enzymes in children with beta-thalassemia major. Pediatr Blood Cancer. 2006;46(7):780-785. doi:10.1002/pbc.20669

13. Al-Hakeim HK, Auda FM, Ali BM. Lack of correlation between nonlabile iron parameters, total carbonyl and malondialdehyde in major thalassemia. J Clin Biochem Nutr. 2014;55(3):203-206. doi:10.3164/ jcbn.14-24

14. Cighetti G, Duca L, Bortone L, et al. Oxidative status and malondialdehyde in beta-thalassaemia patients. Eur J Clin Invest. 2002;32 (Suppl 1):55-60. doi:10.1046/j.1365-2362.2002.0320s1055.x

15. Walter PB, Macklin EA, Porter J, et al. Inflammation and oxidantstress in beta-thalassemia patients treated with iron chelators deferasirox (ICL670) or deferoxamine: an ancillary study of the Novartis CICL670A0107 trial. Haematologica. 2008;93(6):817-825. doi:10.3324/haematol.11755

16. Boudrahem-Addour N, Izem-Meziane $M$, Bouguerra $K$, et al. Oxidative status and plasma lipid profile in $\beta$-Thalassemia patients. Hemoglobin. 2015;39(1):36-41. doi:10.3109/03630269.2014.979997

17. Abduljabbar A, Mehmetçik G. Malondialdehyde and C-Reactive protein values in transfusion dependent thalassemia patients; 2018.

18. Ozturk Z, Genc GE, Kupesiz A, et al. Thalassemia major patients using iron chelators showed a reduced plasma thioredoxin level and reduced thioredoxin reductase activity, despite elevated oxidative stress. Free Radic Res. 2015;49(3):309-316. doi:10.3109/ 10715762.2015.1004327

19. Yanpanitch OU, Hatairaktham S, Charoensakdi R, et al. Treatment of $\beta$-Thalassemia/Hemoglobin $\mathrm{E}$ with antioxidant cocktails results in decreased oxidative stress, increased hemoglobin concentration, and improvement of the hypercoagulable state. Oxid Med Cell Longev. 2015;2015:537954. doi:10.1155/2015/537954

20. Tesoriere L, D'Arpa D, Maggio A, et al. Oxidation resistance of LDL is correlated with vitamin $\mathrm{E}$ status in beta-thalassemia intermedia. Atherosclerosis. 1998;137(2):429-435. doi:10.1016/s0021-9150(97) 00300-6

21. Nasseri E, Mohammadi E, Tamaddoni A, et al. Benefits of curcumin supplementation on antioxidant status in $\beta$-thalassemia major patients: a double-blind randomized controlled clinical trial. Ann Nutr Metab. 2017;71(3-4):136-144. doi:10.1159/000479634

22. Maurya RP, Prajapat MK, Singh VP, et al. Serum malondialdehyde as a biomarker of oxidative stress in patients with primary ocular carcinoma: impact on response to chemotherapy. Clin Ophthalmol. 2021;15:871-879. doi:10.2147/OPTH.S287747

23. Ezzat AM, Abdelmotaleb GS, Shaheen AM, et al. Peroxidative stress and antioxidant enzymes in children with beta-thalassemia major. Med Res J. 2016;15(2):57-62. doi:10.1097/01. MJX.0000511315.53248.78

24. El-Shanshory M, Hablas NM, Shebl Y, et al. Al-hijamah (wet cupping therapy of prophetic medicine) significantly and safely reduces iron overload and oxidative stress in thalassemic children: a novel pilot study. $J$ Blood Med. 2018;9:241-251. doi:10.2147/JBM. S170523

25. Almaiman AA. Proteomic effects of wet cupping (Al-hijamah). Saudi Med J. 2018;39(1):10-16. doi:10.15537/smj.2018.1.21212

26. Ondei L, Estevão I, Rocha MIP, et al. Oxidative stress and antioxidant status in beta-thalassemia heterozygotes. Rev Bras Hematol Hemoter. 2013;35(6):409-413. doi:10.5581/1516-8484.20130122

27. Rasool M, Malik A, Jabbar U, et al. Effect of iron overload on renal functions and oxidative stress in beta thalassemia patients. Saudi Med J. 2016;37(11):1239-1242. doi:10.15537/smj.2016.11.16242 


\section{Publish your work in this journal}

The International Journal of General Medicine is an international, peer-reviewed open-access journal that focuses on general and internal medicine, pathogenesis, epidemiology, diagnosis, monitoring and treatment protocols. The journal is characterized by the rapid reporting of reviews, original research and clinical studies across all disease areas. The manuscript management system is completely online and includes a very quick and fair peer-review system, which is all easy to use. Visit http://www.dovepress.com/ testimonials.php to read real quotes from published authors.

Submit your manuscript here: https://www.dovepress.com/international-journal-of-general-medicine-journal 